\title{
Hepatobiliary and Pancreatic Manifestations of Coronavirus Disease 2019
}

\author{
Vikas Singla ${ }^{1}$ Anil Arora ${ }^{1}$ \\ ${ }^{1}$ Institute of Liver Gastroenterology and Pancreatico Biliary \\ Sciences, Sir Ganga Ram Hospital, New Delhi, India
}

\begin{abstract}
Address for correspondence Vikas Singla, DM, Institute of Liver Gastroenterology and Pancreatico Biliary Sciences, Sir Ganga Ram Hospital, Rajinder Nagar, New Delhi 110060, India (e-mail: singlavikas1979@gmail.com).
\end{abstract}

\begin{abstract}
Coronavirus disease 2019 (COVID-19) is a new infectious disease that has spread rapidly throughout the world. The disease is caused by the severe acute respiratory syndrome coronavirus 2 (SARS-CoV-2), a member of the Coronaviridae family. Though the pulmonary involvement is a major cause of morbidity and mortality, involvement of the gastrointestinal tract, liver, and pancreas has been explained in these patients. The literature is rapidly changing because of influx of new information with every passage of time. The most common hepatic presentation is mild elevation of aspartate transaminase and alanine transaminase, which does not require specific treatment. Occasionally, patients can have severe liver injury. Because of underlying predisposing factors such as diabetes mellitus, hypertension, and obesity, patients with nonalcoholic liver disease may be at risk of severe disease. Patients with decompensated liver disease may also be vulnerable to severe disease. Behavior of SARS-CoV-2 in patients with chronic hepatitis B and C, autoimmune hepatitis, primary sclerosing cholangitis, and primary biliary cirrhosis is yet to be seen. The prevalence and severity of COVID-19 patients with the aforementioned diseases may be different. The effect of

Keywords

- coronavirus disease 2019

- hepatobiliary

- pancreatic

- severe acute respiratory syndrome coronavirus 2 SARS-CoV-2 on an underlying liver disease is not known. COVID-19 may complicate the peritransplant period and throw new challenges in these patients. Drugs used to treat severe COVID-19 may cause liver injury and may have an effect on the underlying disease activity. Both hepatic and pancreatic involvement is related to the severity of COVID-19 disease. Serum amylase and lipase levels may be elevated in patients with severe COVID-19 disease. The involvement of pancreatic islet cells may lead to deranged blood sugar levels and potentially predispose to future diabetes mellitus. There are many unknown facts that will unfold with the passage of time.
\end{abstract}

\section{Overview}

Coronavirus disease 2019 (COVID-19) is an infectious disease caused by severe acute respiratory syndrome coronavirus 2 (SARS-CoV-2) and has spread throughout the globe in a very short span of time, which is beyond the imagination of most of us. The outbreak started in Wuhan, Hubei province of China, in November 2019. The World Health Organization (WHO) declared it as a pandemic on March 12, 2020. As of now, on April 20, 2020, more than 2.4 million patients have

DOI https://doi.org/

10.1055/s-0040-1712079

ISSN 0976-5042. been affected worldwide, with approximately 170,000 deaths across the globe. Risk factors for severe disease and poor outcome are age $>65$ years and underlying comorbid conditions such as cardiovascular disease, diabetes mellitus, hypertension, or chronic obstructive pulmonary disease.

The SARS-CoV-2 virus belongs to the Coronaviridae family of viruses, with a single-strand positive-sense RNA genome. ${ }^{1}$ Viruses of the Coronaviridae family had been identified in various avian and mammalian hosts. ${ }^{2,3}$ Six members of this family are known to cause human disease, and two 
out of these were previously known to cause severe lower respiratory tract infection with high mortality. Severe acute respiratory syndrome coronavirus (SARS-CoV) emerged in southern China in November 2002 and resulted in more than 800 deaths. ${ }^{4}$ The Middle East respiratory syndrome coronavirus (MERS-CoV), first detected in Saudi Arabia in 2012, was responsible for at least 2,220 cases and 790 fatalities, with more than $80 \%$ cases reported in the Middle East and particularly in the Kingdom of Saudi Arabia., ${ }^{5,6}$ Overall damage was limited in both the epidemics. Limited humanto-human transmission of MERS-CoV, and the absence of mild infection and the lack of human-to-human transmission in SARS-CoV virus were the factors responsible for the limited spread. Concerns have been raised previously regarding future serious outbreaks with similar zoonotic viruses. Changes in social practices, the environment, and travel were thought to provide new opportunities for zoonotic pathogens to infect humans. ${ }^{7}$

The fear of pandemic became a truth in late 2019, with Wuhan, Hubei province of China, being the epicenter In late December 2019, clusters of patients were detected with pneumonia of unknown cause, that were epidemiologically linked to a seafood and wet animal wholesale market in Wuhan, Hubei Province, China. Initial research led to novel betacoronavirus belonging to the sarbecovirus subgenus of the Coronaviridae family as the causative agent and was given the name as 2019-nCoV or (SARS-CoV-2). ${ }^{8}$ SARS-CoV-2 is the seventh coronavirus species to cause human disease. ${ }^{1}$

The occurrence of asymptomatic infections and humanto-human transmission are the major factors responsible for failure to contain the virus.

Different coronaviruses bind to different receptors in the human being, such as angiotensin-converting enzyme 2 (ACE2) for SARS-CoV ${ }^{9}$ and CD26 for MERS-CoV. ${ }^{10}$ Receptorbinding domains of SARS-CoV and 2019-nCoV are similar ${ }^{11,12}$ and bind to human ACE2 receptors. ACE2 converts angiotensin II to Angiotensin (1-7). SARS-CoV 2 infection occurs in tissues expressing ACE2 including the lungs, heart, kidneys, brain, liver, and gut. ACE2 is a key enzymatic component of the renin-angiotensin-aldosterone system (RAAS). ACE2 is expressed in both hepatocytes and cholangiocytes. SARS-CoV 2 RNA has been detected in saliva and the stool samples. ${ }^{13}$ The liver may be the site of entry for the gastrointestinal tract.

\section{Hepatic Impairment in COVID-19}

Fever and cough are the most common presentation. Around $20 \%$ of patients can have severe disease and roughly $5 \%$ require intensive care admission. Around 2 to $10 \%$ of patients with COVID-19 had gastrointestinal symptoms such as diarrhea, abdominal pain, and vomiting. ${ }^{14,15}$

Liver impairment has been reported previously with both SARS and MERS-CoV. Liver involvement was seen in $60 \%$ cases of SARS-CoV infection. ${ }^{16,17}$ Hepatic involvement was likely due to the cytopathic effect of the virus, as the reverse transcription-polymerase chain reaction showed SARS-associated coronavirus in liver tissue. Histopathology revealed proliferating cells, apoptosis ballooning of hepatocytes, and mild-to-moderate lobular lymphocytic infiltration. ${ }^{18}$ Similarly, hepatic involvement was reported with MERS CoV infection. ${ }^{19}$

Multiple studies have reported the hepatic involvement in the COVID-19 disease. Liver damage is mostly transient and mild in patients with COVID-19 and can return to normal without any special treatment. Rare cases of severe acute hepatitis have been described in patients with COVID-19. ${ }^{14}$ ACE2 is present in biliary and liver epithelial cells, explaining the involvement of the liver in the COVID-19 disease. ${ }^{20}$ Though ACE2 expression is higher in cholangiocytes than hepatocytes, the most common laboratory abnormality is mild elevation of alanine transaminase and aspartate transaminase, indicating hepatocyte injury. Around 14 to $53 \%$ of cases had elevated alanine aminotransferase and aspartate aminotransferase during disease progression. ${ }^{21}$ Elevated liver enzymes can be due to hepatocyte injury, myositis, myocardial injury, hypoxia, or side effect of various therapeutic agents. Patients with severe COVID-19 seem to have higher rates of liver dysfunction. ${ }^{22}$ Yang et al reported a similar incidence of elevated liver enzymes in survivors and nonsurvivors. ${ }^{23}$ Gamma glutamyl transferase (GGT) and alkaline phosphatase may be elevated in COVID-19 patients. GGT and alkaline phosphatase levels were found to be elevated in $30(54 \%)$ and $1(1.8 \%)$ of 56 patients with COVID-19, respectively.

Hepatic impairment in these patients can be multifactorial. Possible mechanisms are direct cytopathic injury by the virus, immune-mediated injury, cytokine storm, and drug-induced hepatotoxicity.

Exact outcome of COVID-19 in patients with an underlying liver disease is still not known.

Patients with an underlying liver disease may be affected with COVID-19. Whether hepatitis B and C predispose or protect against COVID-19 is unknown. Patients with nonalcoholic fatty liver disease due to underlying hypertension, diabetes mellitus, and obesity may be more prone to the severe disease. As cholangiocytes share the ACE2 receptor, ${ }^{20}$ patients with primary biliary cirrhosis and primary sclerosing cholangitis may have aggravation of biliary injury after the COVID-19. In patients with autoimmune hepatitis who are on steroids and immunosuppression, behavior of COVID-19 is unknown. Whether COVID-19 will be more severe or can lead to a flare-up of autoimmune hepatitis is unknown.

Patients with decompensated liver disease may be more prone to infection by SARS-CoV-2 because of underlying immunocompromised state, and the disease may be severe in these patients.

Many of the drugs used for the treatment of COVID-19 may be hepatotoxic; hence, precautions should be exercised. Therapeutic agents used to manage symptomatic COVID-19, such as remdesivir and tocilizumab, may be hepatotoxic.

COVID-19 infection has been reported in the peritransplant period. In the postoperative period, one patient developed pneumonia followed by elevated liver enzymes, which were thought to be due to graft rejection. The patient was discharged from the hospital. ${ }^{24}$ 


\section{Pancreatic Injury in COVID-19}

Wang et $\mathrm{al}^{25}$ analyzed pancreatic injury in patients with COVID-19. Pancreatic injury was defined as an elevation in pancreatic enzymes. All the patients had underlying viral pneumonia. nine patients had elevated pancreatic enzymes. The authors did not mention about symptoms of pain in these patients. Patients with pancreatic injury were more likely to have anorexia, diarrhea, and severe illness on admission. Direct cytopathic effect and immune-mediated injury have been postulated to be responsible for the injury. The ACE 2 receptor for SARS-CoV has been detected previously in the pancreas, and patients with SARS can have derangement in blood sugar and predisposed to diabetes at follow-up. ${ }^{26}$ Whether SARS-CoV2 can also predispose or worsen diabetes mellitus still needs to be explored.

In conclusion, SARS-CoV2 can cause hepatic and pancreatic injury, which is more common in patients with severe disease. The mechanism of liver injury is likely to be multifactorial, and direct viral effect, immune- and cytokine-mediated injury, and drug-induced injury are the likely possible mechanisms. Patients with decompensated liver disease are likely to have severe disease. Every effort should be made to prevent infection in this highly vulnerable group.

\section{Note}

The work was performed at Sir Ganga Ram Hospital, New Delhi.

\section{Conflict of Interest}

None declared.

\section{References}

1 Su S, Wong G, Shi W, et al. Epidemiology, genetic recombination, and pathogenesis of coronaviruses. Trends Microbiol 2016;24(6):490-502

2 Cavanagh D. Coronavirus avian infectious bronchitis virus. Vet Res 2007;38(2):281-297

3 Ismail MM, Tang AY, Saif YM. Pathogenicity of turkey coronavirus in turkeys and chickens. Avian Dis 2003;47(3):515-522

4 Peiris JS, Yuen KY, Osterhaus AD, Stöhr K. The severe acute respiratory syndrome. N Engl J Med 2003;349(25):2431-2441

5 Zaki AM, van Boheemen S, Bestebroer TM, Osterhaus $A D$, Fouchier RA. Isolation of a novel coronavirus from a man with pneumonia in Saudi Arabia. N Engl J Med 2012;367(19):1814-1820

6 Al-Omari A, Rabaan AA, Salih S, Al-Tawfiq JA, Memish ZA. MERS coronavirus outbreak: implications for emerging viral infections. Diagn Microbiol Infect Dis 2019;93(3):265-285

7 Anderson LJ, Baric RS. Emerging human coronaviruses-disease potential and preparedness. N Engl J Med 2012;367(19):1850-1852

8 Zhu N, Zhang D, Wang W, et al. China Novel Coronavirus Investigating and Research Team A novel coronavirus from patients with pneumonia in china $\mathrm{N}$ Engl J Med 2019;20(382):727-733

9 Li F, Li W, Farzan M, Harrison SC. Structure of SARS coronavirus spike receptor-binding domain complexed with receptor. Science 2005;309(5742):1864-1868
10 Lu G, Hu Y, Wang Q, et al. Molecular basis of binding between novel human coronavirus MERS-CoV and its receptor CD26. Nature 2013;500(7461):227-231

11 Lu R, Zhao X, Li J, et al. Genomic characterisation and epidemiology of 2019 novel coronavirus: implications for virus origins and receptor binding. Lancet 2020;395(10224):565-574

12 Tai W, He L, Zhang X, et al. Characterization of the receptor-binding domain (RBD) of 2019 novel coronavirus: implication for development of RBD protein as a viral attachment inhibitor and vaccine. Cell Mol Immunol 2020;(e-pub ahead of print). doi:10.1038/s41423-020-0400-4

13 Holshue ML, DeBolt C, Lindquist S, et al; Washington State 2019-nCoV Case Investigation Team. First case of 2019 novel coronavirus in the United States. N Engl J Med 2020;382(10):929-936

14 Chen N, Zhou M, Dong X, et al. Epidemiological and clinical characteristics of 99 cases of 2019 novel coronavirus pneumonia in Wuhan, China: a descriptive study. Lancet 2020;395(10223):507-513

15 Wang D, Hu B, Hu C, et al. Clinical characteristics of 138 hospitalized patients with 2019 novel coronavirus-infected pneumonia in Wuhan, China JAMA; 2020 (e-pub ahead of print). doi:10.1001/jama.2020.1585

16 Lee $\mathrm{N}$, Hui $\mathrm{D}, \mathrm{Wu} \mathrm{A}$, et al. A major outbreak of severe acute respiratory syndrome in Hong Kong. N Engl J Med 2003;348(20):1986-1994

17 Tsang KW, Ho PL, Ooi GC, et al. A cluster of cases of severe acute respiratory syndrome in Hong Kong. N Engl J Med 2003;348(20):1977-1985

18 Chau TN, Lee KC, Yao H, et al. SARS-associated viral hepatitis caused by a novel coronavirus: report of three cases. Hepatology 2004;39(2):302-310

19 Alsaad KO, Hajeer AH, Al Balwi M, et al. Histopathology of Middle East respiratory syndrome coronovirus (MERS-CoV) infection - clinicopathological and ultrastructural study. Histopathology 2018;72(3):516-524

20 Chai X, Hu L, Zhang Y, et al. Specific ACE2 expression in cholangiocytesmay causeliver damage after 2019nCoV infection. bioRxiv 2020;(e-pub ahead of print) doi:10.1101/2020.02.03.931766

21 Zhang C, Shi L, Wang FS. Liver injury in COVID-19: management and challenges. Lancet Gastroenterol Hepatol 2020;5(5):428-430

22 Huang C, Wang Y, Li X, et al. Clinical features of patients infected with 2019 novel coronavirus in Wuhan, China. Lancet 2020;395(10223):497-506

23 Yang X, Yu Y, Xu J, et al. Clinical course and outcomes of critically ill patients with SARS-CoV-2 pneumonia in Wuhan, China: a single-centered, retrospective, observational study. Lancet Respir Med 2020;(e-pub ahead of print). doi:10.1016/ S2213-2600(20)30079-5

24 Qin J, Wang H, Qin X, et al. Perioperative presentation of COVID-19 disease in a liver transplant recipient. Hepatology 2020;(e-pub ahead of print). doi:10.1002/hep.31257

25 Wang F, Wang H, Fan J, Zhang Y, Wang H, Zhao Q. Pancreatic injury patterns in patients with COVID-19 pneumonia. Gastroenterology 2020;(e-pub ahead of print). doi:10.1053/j. gastro.2020.03.055

26 Yang JK, Lin SS, Ji XJ, Guo LM. Binding of SARS coronavirus to its receptor damages islets and causes acute diabetes. Acta Diabetol 2010;47(3):193-199 